\title{
Encouraging Initiative in the Classroom with Anonymous Feedback
}

\author{
Tony Bergstrom, Andrew Harris, Karrie Karahalios \\ Department of Computer Science \\ University of Illinois at Urbana-Champaign \\ \{abergst2, harris78, kkarahal\}@illinois.edu
}

\begin{abstract}
Inspiring and maintaining student participation in large classes can be a difficult task. Students benefit from an active experience as it helps them better understand the course material. However, it's easy to stay silent. Opportunities to participate in conversation allow students to question and learn. The Fragmented Social Mirror (FSM) provides students with the ability to anonymously initiate classroom dialog with the lecturer. The system encourages participation by enabling expressive anonymous feedback to reduce evaluation anxiety. The FSM further catalyzes participation by allowing for many simultaneous participants. In this paper, we introduce the FSM as a classroom device, discuss its design, and describe a pilot test of the interface. Initial results indicate a promising direction for future feedback systems.
\end{abstract}

Keywords: Social Mirrors, Classroom, Feedback, Anonymous

\section{Introduction}

Students learn more when they actively engage in the classroom [22]. However the structure of many classes ensures that the lecturer speaks for at least $80 \%$ of the time. Though some students participate, it's expected that five students out of 40 will come to dominate any classroom discussion.

The lecturer's awareness of class comprehension is skewed both by the students' many social pressures and the few speaking opportunities. Students try to present a positive image of themselves to their peers. Thus, they often avoid volunteering information due to evaluation anxiety, a fear of being judged by others for making a mistake or being the focus of attention [22]. It's easy to remain silent. Those students who do speak are generally self-confident or understand the material. However, there is a reluctance to appear too engaged in the classroom. Students who raise the expectations on a group may be ostracized by their peers [17].

In this paper, we present the design of an interface prototype to encourage student engagement and improve the lecturer's awareness in the classroom. The prototype, entitled Fragmented Social Mirror (FSM), aims to create a new communication channel of anonymous dialog between the instructor and the class. Unlike many previous Audience Response Systems [10, 19], FSM allows for expressive text- 
based feedback and may be used throughout the lecture. In our short pilot observation, students in a large class began to initiate interaction with the professor, whereas previously they had only mumbled answers in response to posed questions. In this paper, we describe the design of the FSM in the context of other Audience Response Systems. We also discuss promising initial observations from a classroom pilot study of the FSM.

\section{Feedback in Conversation}

FSM is designed to extend the benefit of backchannel communication. Familiar face-to-face backchannels include "yeahs," "uh huhs," and head nods that show attention to the speaker. Similarly, facial expressions reveal feelings while gestures provide emphasis [15]. From the listener's perspective, these signals show the speaker that the audience is listening and is interested. In a large audience, these visual signals can be lost in the crowd, which necessitates speaking up, murmuring, and applause. Large lectures and speeches require a strong and focused mediation to centralize attention [8]. Without that centralization, small groups of 4-5 individuals form based on proximity of others.

The classroom environment has to balance the need for a strongly focused discussion with the need for student feedback. Lack of participation makes it more difficult to assess the current understanding of students and by not encouraging participation students are less apt to risk being wrong [12]. However, from a constructivist view of learning theory, students need to be actively engaged in their learning. Students learn by actively building their own understanding of new information $[1,21]$. Instruction alone does not directly allow for the construction of knowledge, however effective knowledge construction often adopts a social process between the student and the teacher [7]

Classrooms have addressed this issue by using Audience Response Systems for multiple choice and true/false questions. The interfaces in $[10,19]$ provide a small number of preselected responses of $\mathrm{A} / \mathrm{B} / \mathrm{C} / \mathrm{D}$, and a true/false response. These interfaces are most often used when the lecturer explicitly asks a multiple-choice question of the audience. To be effective, the lecturer has the difficult task of anticipating key moments to query the audience and must specifically structure a lecture to accommodate this new question-answer format. Though each system varies, many include specialized hardware, which are either purchased by or provided for each student $[14,19]$. In the worst cases, when a lecturer does not incorporate interesting interaction into the lecture, the Audience Response Systems can become automated attendance and quiz systems, which students grow to resent [14].

Other feedback modalities such as text-based systems provide opportunities for students to engage with each other $[16,23]$. Studies of dedicated course chatrooms show students will chat about the lecture's content to help explain concepts to confused classmates [23]. However, chat rooms also encourage unrelated discussions, and potentially draw students away from the lecture. Some have gone further and tied the in-class chat discussion to a video recording of the lecture for archival and review [3]. For a practice talk or presentation, these systems allow people to access 
the initial thoughts of the audience in a asynchronous manner. In addition to making help available, this style of active learning helps students communicate concepts to peers for a deeper understanding of the material. Others have brought affective computing to the classroom by using a custom handheld device [4]. This handheld ball can be used to indicate the emotional state of the student to the instructor. Outside of the classroom, text based systems similarly open public dialog into shared events through IRC, instant messaging, Twitter, and Facebook [9, 16, 18]. Though all of these side-channels can contribute to audience discussion, they often leave the speaker out of the loop during the event.

Writing systems, such as Classroom Presenter, benefit from writing as input while still including the instructor in the interaction [2]. A tablet PC system, it allows students to mark directly on the current slide with a stylus, which can then be viewed and shared by students and the instructor. Instructors can set up slides that encourage students to answer questions that can be discussed and reviewed as a group. It also allows for more expressive diagrams, images, and nonverbal communication mechanisms. This method enables a broad sampling of student understanding and encourages active participation with the material.

Related work such as backchan.nl and Conversation Votes create a new feedback channel that integrates approval feedback into group dialog [6, 11]. With backchan.nl audience members organize their collective questions for the speaker in a conference or after a talk. A moderator filters the most appropriate questions from the top rated questions. With Conversation Votes, participants annotated an abstract visualization of conversation with positive and negative votes to highlight agreement during conversation. In small groups, this anonymous feedback increased the level of participation from those less satisfied with previous conversations.

Viewed on an axis of expressivity, distinct categories of low expressivity and high expressivity emerge. Low expressivity systems as in $[6,10,19]$ limit what a student can communicate, but ensure the feedback can be quickly interpreted. High expressivity systems like $[2,11]$ and chatrooms allow students vast communication capabilities, but can require more focused attention for both the lecturer and students.

Our work takes a middle path. FSM provides a meaningful, but constrained, set of signals to be observed alongside the lecture like low expressivity systems, but it allows expressive text to convey personal ideas as in a high expressivity system. As an always-available interface, the FSM captures the fleeting moments of confusion and conveys this information to the lecturer while it can be addressed in context.

\section{The Fragmented Social Mirror}

The Fragmented Social Mirror (FSM) provides feedback based on principles borrowed from our previous work in social mirrors [13]; however, the classroom setting necessitates a break from the standard social mirror design. A social mirror is a realtime depiction of interaction meant to augment natural face-to-face environment. It captures ephemeral moments in conversation and brings them into the public view through visualization. In our previous work, social mirrors displayed abstract visu- 
alizations to depict participation in conversation. The resulting display of conversational dominance, non-participation, and turn taking encouraged more balanced conversation [6]. In these social mirrors, one shared visualization of conversation was projected centrally for all participants to see. On a classroom or large lecture scale, this form does not function as well. There are many more participants involved, and the architecture of the space is different from the spatial layout of small group interaction around one shared table. Furthermore, there is a natural asymmetry in participation due to the lecturer-audience dynamic. This results in less interaction between the lecturer and the audience not suited to the traditional social mirror visualizations.

The term "fragmented" in FSM refers to the use of individual interfaces for each participant as opposed to one shared visualization and the shortened time component as opposed to the full history present in previous social mirrors. In our setup, each participant accesses a Java applet from his or her computer or mobile device, while a large public display is presented to everyone. Furthermore, while a traditional social mirror maintains a persistent history of interaction, FSM highlights questions and comments that are pressing at the specific moment.

\subsection{FSM Design Choices}

The FSM design focuses on capturing and reflecting the unheard and unvoiced dia$\log$ in the classroom. The current design is the result of a long chain of prototypes that sought to balance the need for attention to the interface with attention to the classroom. The final design in this paper served as a culmination of this prototyping, though our pilot demonstrated areas for further refinement such as moderation.

The process of designing the FSM began by observing an active and engaged classroom of $100+$ students to see what students say when engaged in an active class. The lecturers of these classes were generally rated as among the best in the department. They were engaging during lecture and good at encouraging student participation. To facilitate more participation the lecturers posed a question and waited for responses - thus guaranteeing an answer or a question for clarification. We noted all the types of student responses to better understand what a student wants to say during class. The responses were narrowed down to the following list of categorical responses:

- Questions: Students provide new questions based on what has just been taught.

- Information: Students add their own connection to outside subjects.

- Agreement/Disagreement: Answering a Lecturer's question.

- Slow Down/Redo: Students did not understand the lecturer.

- Cannot Hear/Repeat: Students did not hear the lecturer.

Our list was very similar to feedback available in other work to mark up a presentation slide [20]. We began to investigate this set of six messages for our prototypes. These six messages would serve as categories with the ability for students to include a short text message for explanation. The message categories serve as a means to 
identify and group similar responses and highlight important categories like questions. In parallel with our interface design, we investigated imagery for each of these six categories of messages (described in the next section). Due to this process, "Slow Down/Redo" and "Cannot Hear/Repeat" were eliminated. Suitable icons could not be found and they easily be replaced by an "Information" message with appropriate text.

Many of our initial interface prototypes borrowed design components from the Conversation Clock and Conversation Votes [5, 6], they incorporated the feedback into a timeline that structured the activity throughout the session. In some cases, we included indications of speaker. Much like a standard instant messenger, the full history of messages could be read through at any time. These interfaces showed potential for the review of archival classroom data, but did not serve our purpose of encouraging classroom interaction. These prototypes, tested amongst our own group, required too much attention to adequately understand.

After refining the initial prototypes, we settled on a simple interface students could use without pulling their attention too far from the lecturer. Thus the input of the FSM was used only for capturing one comment. The history of feedback was only seen on the public display and limited that history to the most recent comments. Additionally, the needs of the lecturer necessitated this type of design. The lecturer needed to be able to read feedback from the hundreds in the audience while still being able to teach effectively. In past studies, a social mirror was primarily viewed by the listeners (and not the speaker) in conversation because they had more free attention [5]. In this design, the captured feedback of conversation is significantly pared down, so that the lecturer can receive the benefits from the social mirror with minimal attention. Therefore, current comments/questions are displayed so as not to overwhelm the viewers with a long history.

\section{Iconographic Messages}

The FSM interface passes information through icons. These graphics serve to simplify the message so that the lecturer might easily understand the classroom without reading too much content. Based on informal observation of classroom sessions and prior work [20], we designed icons based on the messages earlier: "I have a question," "I have information/an answer," "Yes/agree," "No/disagree," "Speak Up," "Slow Down." Three researchers independently drew any graphic that they felt reasonably captured these messages. We combined them into sets for each category, with a total of 5-15 images for each message.

We conducted a survey of Computer Science undergraduates to test our icon designs. A total of 54 Computer Science undergraduates completed our survey. Their feedback identified 17 icons that convey the intended message. Figure 1 shows all 17 icons. None of the icons for "Slow Down" conveyed an adequate message to the student. We eliminated this message, as well as the "Speak Up" messages in favor of a simpler 4-icon interface. Students can use the Information and Question messages with additional text to signal "Slow Down" and "Speak Up." 
The icon represents confusion or the desire to ask a question.
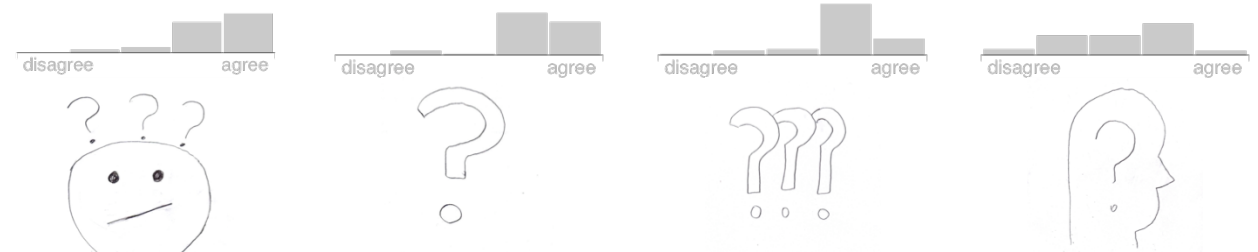

The icon represents an audience member has new information.
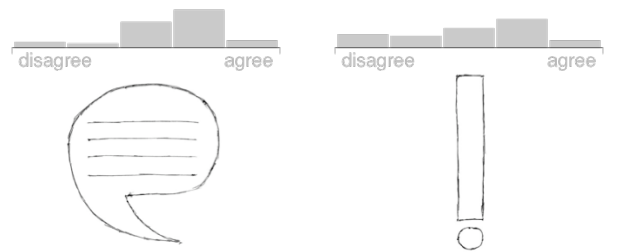

The icon represents disagreement or a negative response.
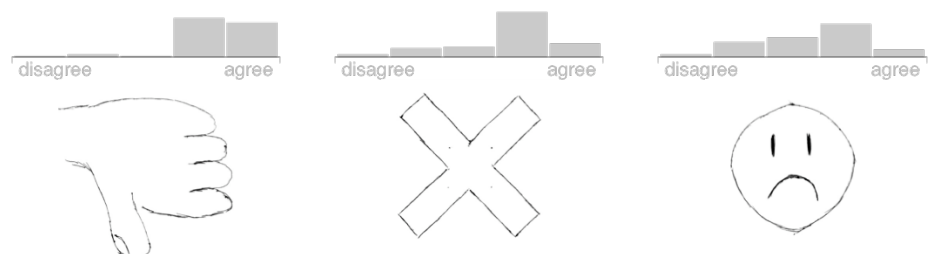

The icon represents agreement or a positive response.
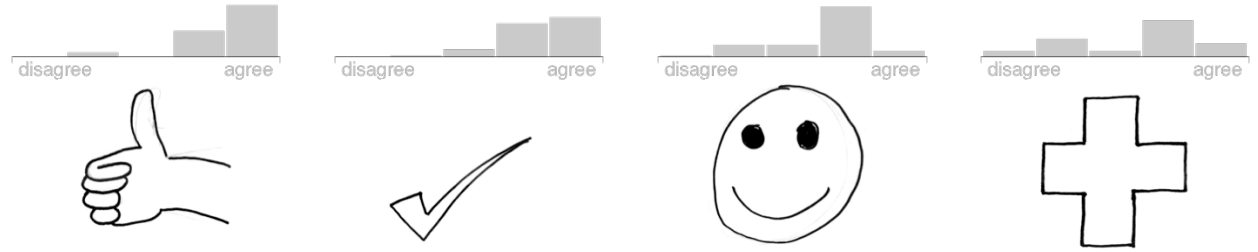

The icon represents that the lecturer needs to increase their volume.
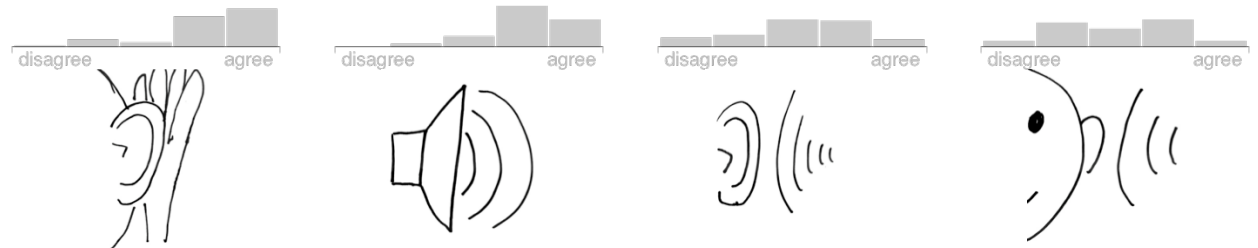

Fig. 1. The results of 54 individuals rating our sets of icons produced these icons as the most indicative of their intended messages. The image survey informed our final selection of icons seen in the interface (Figures 1 and 3). One other message, to indicate the speaker was moving too fast, produced no positively rated icons. 


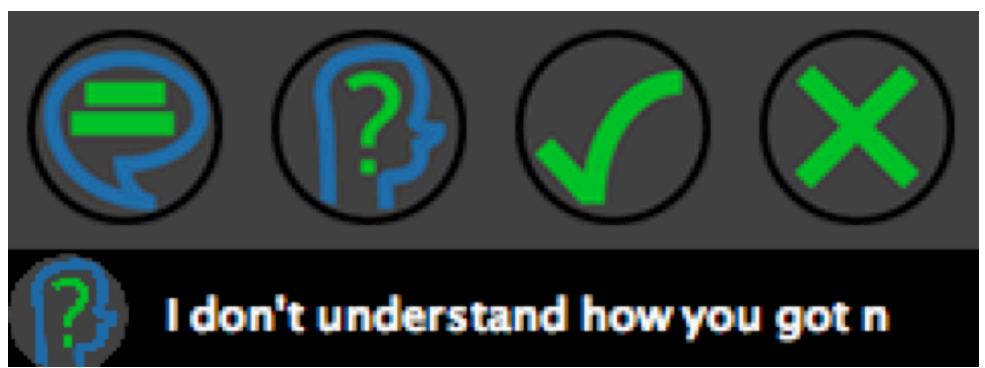

Fig. 2. The input device is small and simple for classroom use. The two left icons for information and questions allow for typing phrases to send along with the icon message.

\section{FSM Interfaces}

There are two FSM interfaces - the student's client interface for a computer or handheld device (Figure 2) and a larger public screen for the lecturer and audience (Figure 3). The public display is situated in the front of the room, though the lecturer sees the public display on a personal screen. The four different preselected icons categorize student responses in the student interface. The icons represent: Information, Questions, yes/agree, no/disagree. Of the four categories or signals, the Information and Question signals can be augmented by a 40-character message. The short messages allow students to clarify their questions or possible answers when there is no opportunity to speak while the yes/no buttons allows students to answer simple questions quickly.

Students use the client interface in Figure 2 to send their message to the public display shown in Figure 3. All messages on the public display are grouped by their associated icon to increase legibility for the speaker. The speaker can look up and see many questions that need to be addressed or they can glance over answers that

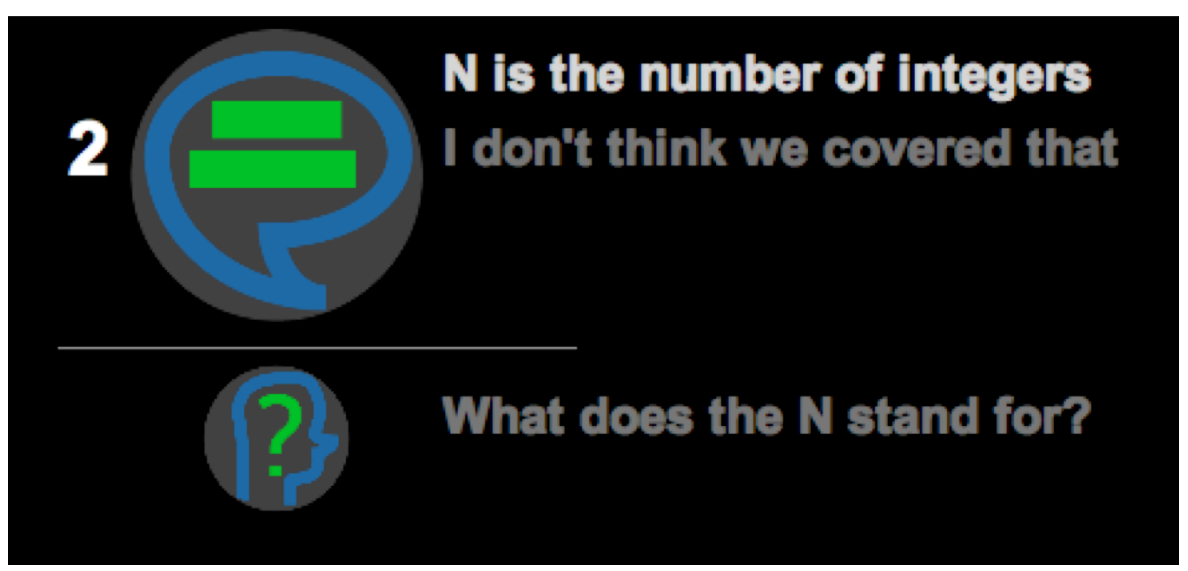

Fig. 3. The public display groups messages by icon and highlights the most recent feedback from the class. To the left of each icon, a counter indicates multiple messages of the same type, particularly useful when using the yes/no feedback buttons. 
students provided via the display. The icon group with the most messages moves to the top of the screen with a larger icon. The most recent message of this icon appears at the top of that icon in white text set against the black background. As a message ages, it fades to grey before finally disappearing after a pre-configured time. For icons with multiple messages, a count is displayed to the left of the icon.

Messages on the public display are limited to recent messages. Only the most recent minute of activity is visible; each message fades in brightness over the minute before disappearing from view. The rationale for this design was two fold: (1) we did not want the lecturers to be confused or overwhelmed by reading old questions from a prior part of the lecture and (2) if a question goes unanswered and disappears, this removal may encourage a student to verbalize the question in class or to repost it. One of our main goals is to encourage more class interaction. If a student can "see" that they are not alone in their confusion, they may be less apprehensive to speak out and ask a question.

Once a student sends a signal via posting an icon, they are blocked from sending additional signals for a brief period (10 seconds in our pilot) to discourage excessive social chatter and monopolization of the channel. While there is some room for abuse as with the backchan.nl system, where some users voted up questions for humor [11], the public availability of the channel is ultimately at the discretion of the lecturer.

\section{Pilot Study}

\begin{tabular}{cccc} 
Session & Students & Computers & On FSM \\
\hline 1 & 108 & 17 & \\
2 & 125 & 25 & \\
3 & 112 & 19 & \\
\hline 4 & 102 & 23 & 14 \\
5 & 80 & 19 & 15 \\
6 & 73 & 14 & 5
\end{tabular}

Fig. 4. This table shows the number of people in the classroom for each session as well as the number of computer visible. Though we did request students to bring their computers to the sessions with the Fragmented Social Mirror. The number of computers remained essentially unchanged.

We conducted a pilot study to investigate the FSM in the classroom. We began by observing the participation levels before the introduction of the FSM and again with the FSM in place. For this, we observed a required second year course with roughly 180 registered students at the beginning of the semester. The instructor was not affiliated with our research team. We observed a total of six course sessions: three initially without any augmentation, and three with the addition of the FSM. During observation, an average of 100.0 students were in attendance, though there were 
fewer students in the final sessions (attributed to an intervening midterm and final day to drop the course). Given the large class, not many students had the opportunity to speak, and most did not. A summary of the attendance is visible in Figure 4.

\section{Preliminary Survey Responses and Questions}

\section{in Class}

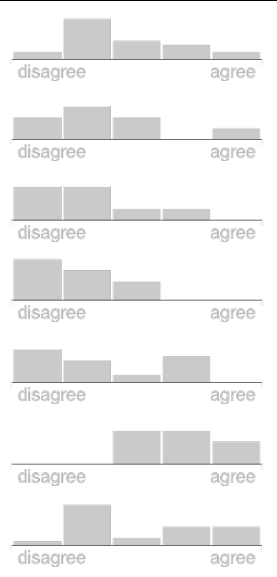

\section{$\frac{1+\square}{\text { disagree }}$}

\section{Satisfaction}

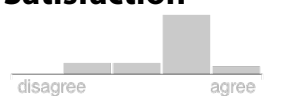

Ambivalence

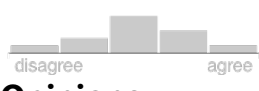

Opinions

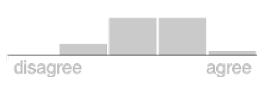

in Discussion

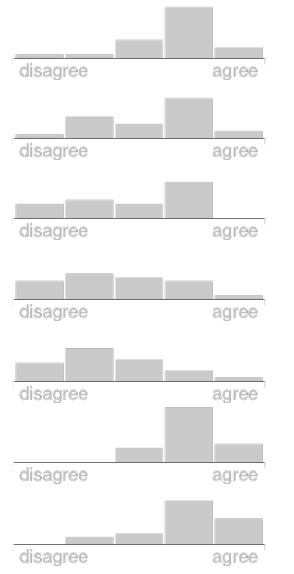

Dissatisfaction

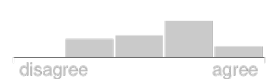

Enthusiasm

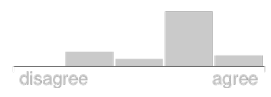

Questions

\section{Question}

I participate.**

I participate more than once.**

I ask questions.*

I ask multiple questions. ${ }^{* *}$

I have more questions than time allows.

I feel my questions are respected.

I am comfortable asking ques-

tions. ${ }^{* *}$

I am comfortable asking for clarifications. ${ }^{* *}$

I am comfortable expressing satisfaction/dissatisfaction.

I am comfortable expressing ambivalence/enthusiasm.*

My peers are comfortable expressing opinion/questions.

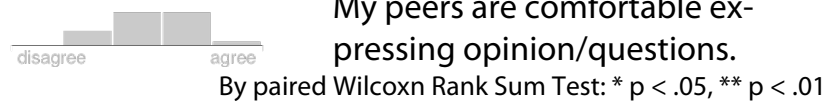

Fig. 5. Students reported they were uncomfortable asking questions in class, though it was less the case in smaller recitation sections. They are aware that they do not participate in class and are less likely to ask questions even though they have them. There is reluctance amongst the students to speak up and participate. Survey with 23 respondents. All graphs have the same scale. 
Prior to testing the FSM in class, we sent a pre-survey and described the use of the FSM. The survey inquired about the student's comfort level while participating in class versus their smaller discussion sections. Feedback from the survey confirmed that students are not comfortable asking questions or asking for clarification during class, though they are more comfortable asking in their smaller recitation sections. Similarly, they recognize that they do not participate or ask questions during class (Figure 5).

Our initial observations showed little interaction between audience and lecturer over the course of three 50-minute sessions. The only activity from the audience was in response to questions posed by the lecturer. For example, students were asked "n is divisible by what?" and "What is the cardinality of set Q?" in reference to a proof. The class averaged about four responses per class. The students initiated zero interactions themselves, five of the twelve responses were general indefinite murmurs from the class, and two responses involved raising hands. Various sets of 1-3 unidentified students spoke up to answer the remaining six questions.

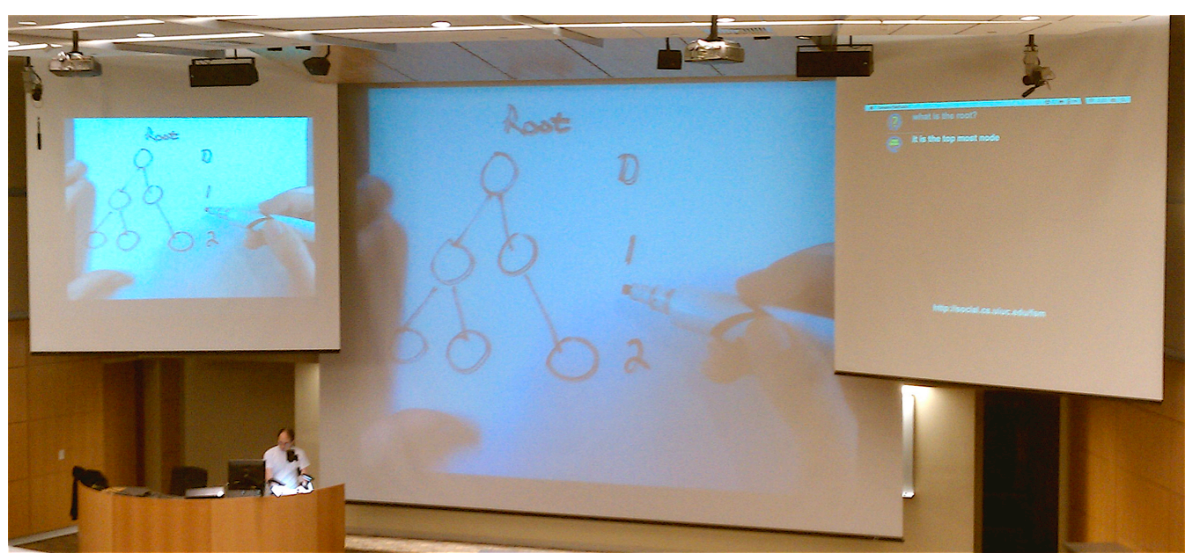

Fig. 6. We tested the Fragmented Social Mirror in a large lecture hall with three projection screens. Typically, the instructor repeated the same material on all three screens. For the study, the Fragmented Social Mirror replaced one screen during class time. The screen by the lecturer also displayed the public interface.

We tested the FSM in three class sessions and found the students were proactive in using the system. In the classroom, the lecturer used a central projection screen to work through problems by hand while a smaller screen displayed the public display to the right of the larger screen (Figure 6). At the lecture podium, the lecturer also had a copy of the public display available during the class activity.

With the system in place, Students initiated dialog with the lecturer by asking questions 11 times, compared to zero without the system. When on topic, students used the Fragmented Social Mirror to ask questions of the professor, keep the professor from moving on too quickly, and to answer any questions the professor posed. Figure 7 summarizes the participation in each of the 6 classes. Most of the on-topic dialogs either began with or contained a question for the instructor. They lead to discussions with the instructor and information to enrich the class. However, there 
were also many off-topic messages. These messages were irrelevant to the class topic and were used to draw the attention of other classmates away from the lecture material for their own entertainment.

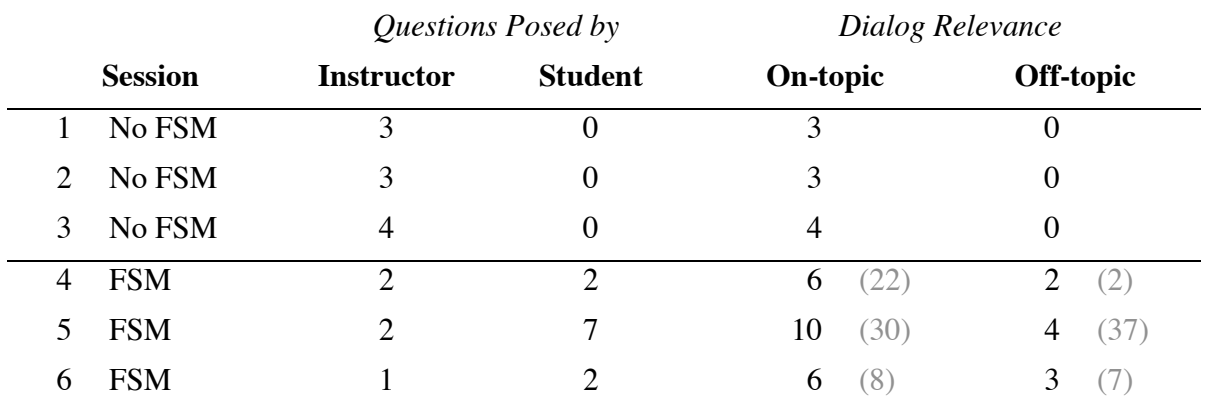

Fig. 7. The Fragmented Social Mirror encouraged students to initiate questions and dialog with the instructor. Many dialogs began with or contained questions, though some were responses to indicate comprehension of the material. The anonymity of the interface also encourages unrelated dialog in class. A dialog in the Fragmented Social Mirror could include multiple messages - thus, \# dialogs (\# messages) includes both the count of interaction instances and total messages.

\section{Example FSM Dialogs}

Excerpts from the FSM sessions appear below. In this first example, students requested information that the professor was not trying to teach but established an interesting aside on history related to the lesson:

Instructor [discussing the Karatsuba Algorithm]

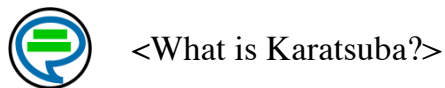

Instructor

Karatsuba is the guy who invented it, Anatolii Karatsuba. [Instructor continues with a bit more history.] 
As another example, the student's lack of understanding prompts him or her to ask for clarification on calculating tree height.

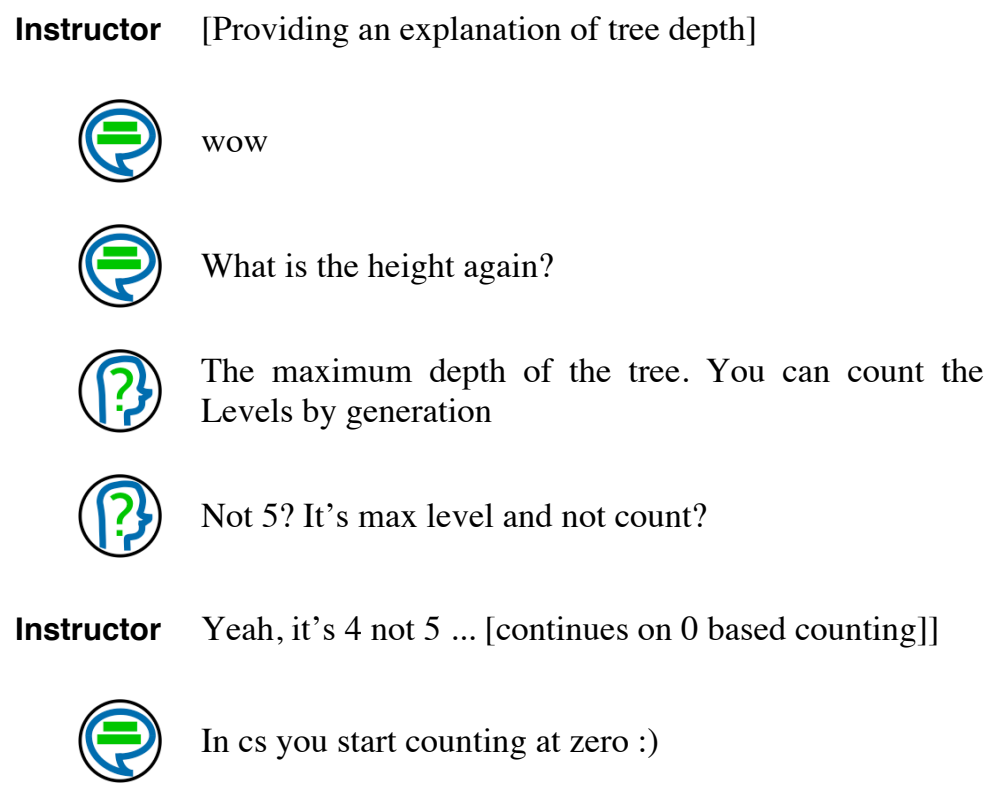

However, with the addition of initiating comments, there was also an increase in comments solely intended to draw attention away from lecture. These messages often had nothing to do with the lecture or a question tended to come in bursts in order to overwhelm the public display for a short time. As an example of such a burst:

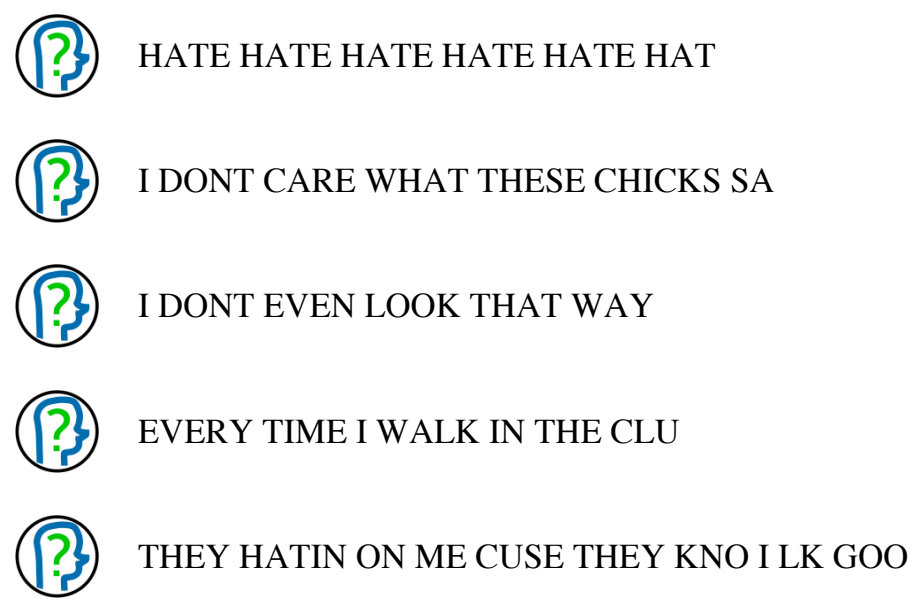


This type of interaction was most prevalent in the second session. The lecturer was inclined to read them, see that they were not relevant and either laugh, if it were funny, or state "I don't know what this means." However, the increase of messages also meant that the lecturer was more likely to miss relevant exchanges where a student was asking for help:

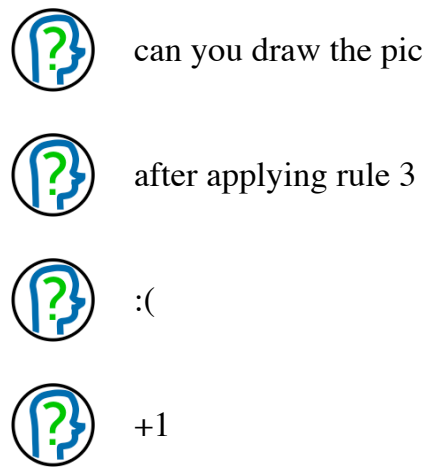

\section{After the sessions}

We had only planned to gather initial observations to refine the system in these first sessions; however, the instructor was excited to see the students participating and invited us to return with the system for further studies. After the lectures, she indicated that it's always been hard to get this many students to say anything, even with encouragement. The simplicity of the display was also deemed useful, as she could read the questions with a glance. Additionally, the asynchronous nature allowed students to ask their questions while she was still explaining - thus allowing her to work the question into that explanation or come back to it later. Student feedback indicated the device was useful as they "didn't have to try to get the professors attention" by raising a hand from the back of the lecture.

Students also saw the benefit of the interface, and felt it was easier to participate in the classroom (Figure 8). However, they recognized the difficulty of maintaining order in the anonymous display and provided suggestions to keep the interface on topic. One such suggestion was to make the display semi-anonymous; implement a publicly anonymous interface that retains the identity on the lecturer's display. In this way the instructor could call out any abuse of the display, while protecting the identity of any others who were uncomfortable commenting in front of the class. A similar suggestion would simply log the identities for review after class.

\section{Discussion}

The Fragmented Social mirror touched the surface of integrated feedback in large discussions by allowing a controlled set of feedback that could express a student's question or response. As we mentioned in related work, the field can be divided into 
the high expressivity interfaces that require more attention and the low expressivity interfaces that do not allow students to indicate their questions.

In the sessions with the FSM, the classroom dialog was more involved. The lecturer felt like she was talking to people rather than at people while the students took a more proactive role in directing conversation to points that were not understandable. With 100 students, evaluation anxiety limits the individuals willing to speak however we have shown that anonymous feedback can break the barrier and include more students.

Response

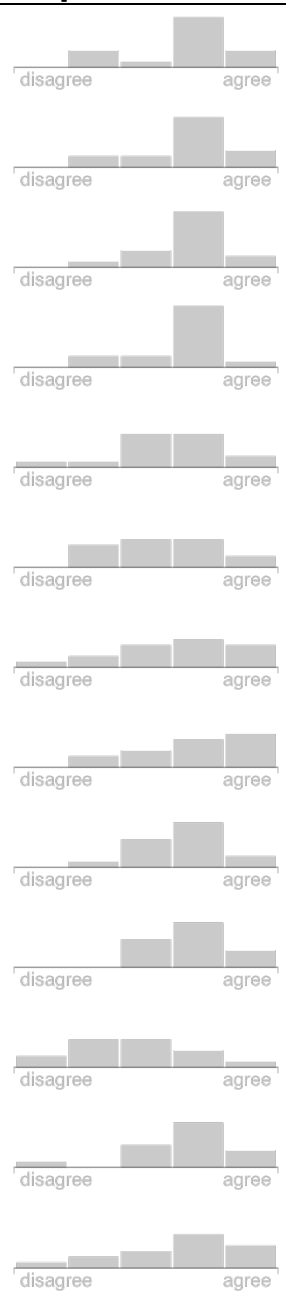

\section{Questions}

The system encourages participation.

The system encourages my own participation.

The system encouraged me to ask questions.

The system encouraged my peers to ask questions.

The system made me feel connected to the lecture.

The system made me feel connected to the presenter.

The system made me feel connected to the other audience members.

The icons were clear in their meaning.

The use of text entry was clear in it's meaning.

The system was easy to use.

The feedback from others helped me to understand the lecture material.

The feedback from others made the lecture more enjoyable.

The system is a worthwhile addition to the lecture and discussions.

Fig. 8. Students reported the Fragmented Social Mirror encouraged participation and connected them to the lecture. Students reported the lectures being more enjoyable and saw the feedback as a worthwhile addition to the lectures. 
Our interface was not perfect; the classroom sessions revealed that while anonymity opens opportunities for discussion, it must be tempered in some manner. In both the second and third sessions, some individuals engaged in the public conversation by adding potentially disruptive comments that lead to legitimate questions not being discussed. As a prototype, we did not fully flesh out any mechanisms to prevent this type of interaction. Perhaps the lecturer should be able to flag such comments during conversation to lock out individuals, or the lecturer should be able to identify individuals after the classroom session and deduct points in some manner. While it is tempting to simply allow the class to moderate itself, the design must be careful not to become more of a distraction as it requires interaction outside the scope of a learning task and draws them away from the lecture.

The classroom sessions also made it apparent that the positive and negative responses should redesigned to provide more flexibility. Though students did use them as feedback for the lecturer, they also use the agreement checkmark to indicate a "me too" when other students raised a question. Others adopted a convention of adding a " +1 " as seen in the example conversations. A revamped system might allow a student to indicate "me too" and ensure the question stays visible long enough for the lecturer to see the question.

Our surveys underscore the need for large classrooms to tap into technological backchannels. Students know that they do not participate in large classroom settings even though they have questions. They are not comfortable asking questions in such a large group. The survey after the use of our system shows that students felt encouraged themselves and the class to ask questions, they found it to make the lectures more enjoyable, and it was a worthwhile addition to the lecture.

The FSM interface received a positive response from both students and lecturer. Our initial study highlights the use of anonymous signals in large classroom has potential to draw in more active participation of the students and audience.

\section{Conclusion and Future Work}

A large audience automatically reduces the possibilities for participation in a lecture setting. Rather than accept this and move on, technology has provided new channels to engage students throughout the lecture.

Our own work shows that expressive feedback can be gathered from lightweight systems. The use of these channels was shown to increase engagement, to benefit the students, and to spark initiative where there was none before.

While our system was successful in engaging students and encouraging participation, we acknowledge it is not a definitive study and has limitations. Our study was a small study to test our conception of anonymous feedback. The system, with refinements, should be further tested over a longer term and in multiple classrooms. Many untested facets of the interface can be further explored. We advocate anonymous feedback based on the premise of evaluation anxiety, though we have not yet tested the effects of allowing or enforcing identity in the FSM. 
The Fragmented Social Mirror indicates that the use of text based anonymous feedback has potential for promoting engagement in the classroom. A long term study could investigate the effects on learning outcomes: does the FSM encourage students who are already engaged in class to further surpass their peers, or does it genuinely help students who just need a small boost to get involved?

We hope to explore these further questions on the benefits of classroom feedback in future works with the FSM and other interfaces.

\section{References}

1. Anderson, C. W.. Strategic teaching in science. In Strategic teaching and learning: Cognitive instruction in the content Areas, B. F. Jones, A. S. Palincsar, D. S. Ogle, and E. G. Carr, Eds 1987.

2. Anderson, R., Anderson, R., Davis, P., Linnell, N., Prince, C., Razmov, V., and Videon, F. Classroom Presenter: Enhancing Interactive Education with Digital Ink. Computer. 40(9):56-61. 2007.

3. Baecker, R., Fono, D., Lillian, B., and Collins, C. Webcasting made interactive: persistent chat for text dialogue during and and about learning events. Proceedings of the 2007 conference on Human interface: Part II. 2007.

4. Balaam, M., Fitzpatrick, G., Good, J., and Luckin, R. Exploring affective technologies for the classroom with the subtle stone Proceedings of the 28th international conference on Human factors in computing systems. CHI '10. 2010.

5. Bergstrom, T. and Karahalios, K. Seeing More: Visualizing Audio Cues. Proceedings of Interact. 2007.

6. Bergstrom, T. and Karahalios, K. Vote and Be Heard: Adding Back-Channel Cues to Social Mirrors. Proceedings of Interact. 2009.

7. Brown, J., Collins, A. M., and Duguid, P. Situated cognition and the culture of learning. 1989.

8. Dunbar, R. I. M., Duncan, N. D. C., and Nettle, D. Size and structure of freelyforming conversational groups. Human Nature. 6(1). 1995.

9. Ebner, M. and Reinhardt, W. Social networking in scientific conferences - Twitter as tool for strengthen a scientific community. In Proceedings of the 5th EduMedia Conference. 2009.

10. Fitch, J. L. Student feedback in the college classroom: A technology solution. Educational Technology Research and Development. 52(1):71-77. 2004.

11. Harry, D., Green, J., and Donath, J. Backchan.nl: integrating backchannels in physical space. Proc. of CHI. 2009.

12. Jones, M. G. and Gerig, T. M. Silent Sixth-Grade Students: Characteristics, Achievement, and Teacher Expectations. The Elementary School Journal. 95(21994.

13. Karahalios, K. and Bergstrom, T. Social Mirrors as Social Signals: Transforming Audio into Graphics. IEEE Computer Graphics and Applications. 29(5):22-32. 2009.

14. Kay, R. H. and LeSage, A. Examining the benefits and challenges of using audience response systems: A review of the literature. Comput. Educ. 53(3):819-827. 2009.

15. Krauss, R. M., Garlock, C. M., Bricker, P. D., and McMahon, L. E. The role of audible and visible back-channel responses in interpersonal communication. Journal of Personality and Social Psychology. 35(7):523-529. 1977.

16. McCarthy, J. F. and boyd, d. m. Digital backchannels in shared physical spaces: experiences at an academic conference CHI '05: CHI '05 extended abstracts on Human factors in computing systems. 1641-1644. 2005. 
17. Parks, C. D. and Stone, A. B. The desire to expel unselfish members from the group. Journal of Personality and Social Psychology. 99(2):303-310. 2010.

18. Shamma, D. A., Kennedy, L., and Churchill, E. F. Tweet the debates: understanding community annotation of uncollected sources WSM '09: Proceedings of the first SIGMM workshop on Social media. 3-10. 2009.

19. Stowell, J. R. and Nelson, J. M. Benefits of Electronic Audience Response Systems on Student Participation, Learning, and Emotion. Teaching of Psychology. 34(4):253-258. 2007.

20. VanDeGrift, T., Wolfman, S. A., Yasuhara, K., and Anderson, R. J. Promoting Interaction in Large Classes with a Computer-Mediated Feedback System. University of Washington, Computer Science and Engineering. 2002.

21. von Glasersfeld, E. Cognition, Construction of Knowledge, and Teaching. History, Philosophy, and Science Teaching. 80(1):121-140. 1989.

22. Weaver, R. R. and Qi, J. Classroom Organization and Participation: College Students' Perceptions. The Journal of Higher Education. 76(5):570-601. 2005.

23. Yardi, S. Whispers in the Classroom. In Digital Youth, Innovation, and the Unexpected, T. McPherson,Ed 2008. 